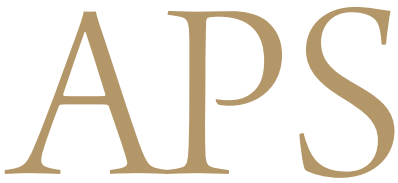

Archives of Plastic Surgery

\title{
Medial and Lateral Canthal Reconstruction with an Orbicularis Oculi Myocutaneous Island Flap
}

\author{
Jihyeon Han ${ }^{1,2}$, Sung Tack Kwon ${ }^{1}$, Suk Wha Kim ${ }^{1}$, Eui Cheol Jeong ${ }^{1,2}$ \\ ${ }^{1}$ Department of Plastic and Reconstructive Surgery, Seoul National University College of Medicine, Seoul; ${ }^{2}$ Department of Plastic Surgery, \\ SMG-SNU Boramae Medical Center, Seoul, Korea
}

Background The eyelid and canthal areas are common locations for cutaneous tumors. The medial canthus includes, among many other apparatuses, the canthal tendon and lacrimal canaliculi, and its characteristic thin and supple skin is hard to mimic and restore using tissue from other regions. Accordingly, reconstruction of the canthal area can prove challenging for surgeons. Although various methods, such as skin grafts and local flaps from adjacent regions, have been utilized for reconstructive purposes, they present known disadvantages. However, we were able to successfully reconstruct both lateral and medial canthal area defects by using orbicularis oculi myocutaneous island flaps.

Methods Our study included seven patients who underwent medial or lateral canthal region reconstruction, using orbicularis oculi myocutaneous island flaps, between 2011 and 2014, following either cutaneous tumor excision or traumatic avulsion injury.

Results Five patients had basal cell carcinoma, one had squamous cell carcinoma of the eyelid, and one had sustained a traumatic avulsion injury of the eyelid and canthal area. Entire flap loss was not observed in any patient, but one-a heavy smoker-showed partial flap loss, which healed with secondary intention and yielded acceptable results. Donor site morbidity was not observed, and all patients were satisfied with their surgical outcomes.

Conclusions The canthal regions can be successfully reconstructed with orbicularis oculi myocutaneous island flaps. These flaps offer several key advantages, including similarity in texture, color, and thickness to the recipient site and a negligible incidence of donor site morbidity.

\section{Keywords Eyelids / Carcinoma / Myocutaneous flaps}

\author{
Correspondence: Eui Cheol Jeong \\ Department of Plastic and \\ Reconstructive Surgery, Seoul \\ National University College of \\ Medicine, Department of Plastic \\ Surgery, SMG-SNU Boramae Medical \\ Center, 20 Boramae-ro 5-gil, \\ Dongjak-gu, Seoul 156-707, Korea \\ Tel: +82-2-870-2331 \\ Fax: $+82-2-831-2826$ \\ E-mail: ejeong.md@gmail.com
}

No potential conflict of interest relevant to this article was reported.

Received: 27 May 2014 • Revised: 16 Aug 2014 • Accepted: 18 Aug 2014

pISSN: 2234-6163 • elSSN: 2234-6171 • http://dx.doi.org/10.5999/aps.2015.42.1.40 • Arch Plast Surg 2015;42:40-45

\section{INTRODUCTION}

The eyelid and canthal areas are common locations for malignant skin tumors. The medial canthal region includes the medial canthal tendon, lacrimal apparatus, and neurovascular structures from the deep orbit [1]. The skin covering the canthal region is thin and supple in character, and expresses the curvature of the periorbital area. In addition, the intactness of both the lateral and medial canthal regions is crucial to the shape and appearance of the eye, and even the slightest deformity or asymmetry is noticeable. The complexities of the region's structural anatomy, thus, make reconstruction after skin cancer resection challenging for surgeons. Furthermore, traditional methods have failed to restore the color, thickness, and consistency of the 
skin, leading to an unnatural appearance.

The authors present their experience using orbicularis oculi myocutaneous island flaps to perform medial and lateral canthal region reconstruction in seven patients. Entire flap loss did not occur in any of the cases, and the results for all patients were aesthetically acceptable, without any functional problems of the eyelid aperture. The authors believe that this technique can provide a reliable alternative to resurfacing the canthal regions, especially in elderly patients with redundant upper eyelid skin.

\section{METHODS}

\section{Study subjects}

Between 2011 and 2014, we performed cutaneous tumor excision and reconstruction of the medial or lateral canthal region in 6 patients, and reconstruction alone in 1 patient who had sustained a traumatic avulsion injury (Table 1). All these patients underwent either medial or lateral canthal reconstruction, using the orbicularis oculi myocutaneous island flap technique.

\section{Operative procedure}

When employing the orbicularis oculi myocutaneous island flap technique, the size of the patient's canthal area defect is first measured after resection. An appropriately sized flap is then marked on the upper eyelid, similar to the upper blepharoplasty design used in the surgical treatment of dermatochalasis. Next, the flap's lower margin is placed either on the innate supratarsal fold or, in cases without a fold, at $7 \mathrm{~mm}$ above the eyelid margin. If the defect is in the medial canthal area, a medially based flap is used; if it is in the lateral canthal area, a laterally based flap is used. The flap is then elevated to approximately $4 \mathrm{~mm}$ from the canthal angle, to include both the skin and the orbicularis oculi muscle. Care must be taken at this point to avoid detaching the orbicularis oculi muscle from the bed, so that the muscular perforator of the peripheral arcade may be preserved as the pedicle. Once it is elevated, the flap is rotated on its pedicle and, if neces- sary, passed through a subdermal tunnel. The flap is then inset after it is tailored to fit the shape of the defect. Finally, the donor site is approximated and closed primarily, as in an upper blepharoplasty (Fig. 1).

\section{Case 1}

A 59-year-old man presented with a biopsy-proven basal cell carcinoma involving the left lower eyelid and medial canthus. The carcinoma had first occurred 1 year ago, in the form of a pigmented papule. The patient underwent excision with a 3-mm margin, followed by reconstruction with a 4-cm-long and 6-mm-wide, medially based orbicularis oculi myocutaneous island flap from the left upper eyelid. The flap was transferred to the site of the defect (measuring $1 \times 0.9 \mathrm{~cm}$ ) through a subdermal tunnel in the medial canthal area after partial deepithelialization. The punctum was found to be involved, and an ophthalmologist subsequently performed lacrimal duct reconstruction, using a silicone tube. Lateral tarsorrhaphy was maintained until postoperative day 6 (Fig. 2).

\section{Case 2}

A 54-year-old man presented with a biopsy-proven basal cell carcinoma on the right lower eyelid. The lesion had first occurred 6 years previously and had been slowly growing in size. At the time of the patient's visit, the nodule measured $1 \times 0.8 \mathrm{~cm}$. He subsequently underwent excision with a 4-mm margin, followed by reconstruction with a $50 \times 7 \mathrm{~mm}$, laterally based orbicularis oculi myocutaneous island flap from the ipsilateral upper eyelid. The flap was delivered through a subdermal tunnel to the resection site, and was inset after it was tailored. Temporary tarsorrhaphy was maintained for 5 postoperative days (Fig. 3).

\section{RESULTS}

In all seven cases, the patients' hospital course was uneventful, and their reconstructed flaps were viable with no incidence of Table 1. Summary of patients $(n=7)$

\begin{tabular}{|c|c|c|c|c|c|c|}
\hline Case & Sex & Age (yr) & Diagnosis & $\begin{array}{c}\text { Flap size } \\
\text { (defect size, } \mathrm{mm} \text { ) }\end{array}$ & $\begin{array}{l}\text { Follow-up } \\
\text { period (mo) }\end{array}$ & Complication \\
\hline 1 & Female & 81 & BCC, left lower eyelid and medial canthal area & $13 \times 10$ & 23 & - \\
\hline 2 & Female & 81 & SCC, left lower eyelid and lateral canthal area & $20 \times 17$ & 10 & - \\
\hline 3 & Female & 53 & BCC, left upper eyelid and medial canthal area & $13 \times 12$ & 11 & - \\
\hline 4 & Male & 43 & Avulsion, right lower eyelid and lateral canthal area & $20 \times 10$ & 7 & - \\
\hline 5 & Male & 66 & BCC, left medial canthal and nasal area & $19 \times 18$ & 12 & Partial necrosis of the flap \\
\hline 6 & Male & 59 & BCC, left lower eyelid and medial canthal area & $10 \times 9$ & 8 & - \\
\hline 7 & Male & 54 & $\mathrm{BCC}$, right lower eyelid and lateral canthal area & $50 \times 7$ & 7 & - \\
\hline
\end{tabular}




\section{Fig. 1. Elevation of the orbicularis oculi myocutaneous island flap}

(A) Elevation of a laterally based orbicularis oculi myocutaneous flap to cover defects in the lateral lower eyelid or lateral canthal area. The musculocutaneous perforator, indicated with a dotted line, enters at an approximately 4-mm horizontal distance from the lateral canthus. The muscle pedicle is 1-cm wide. (B) Elevation of a medially based orbicularis oculi myocutaneous flap to cover the defects in the medial lower eyelid or medial canthal area. The musculocutaneous perforator, indicated with a dotted line, enters at an approximately 4-mm horizontal distance from the medial canthus.
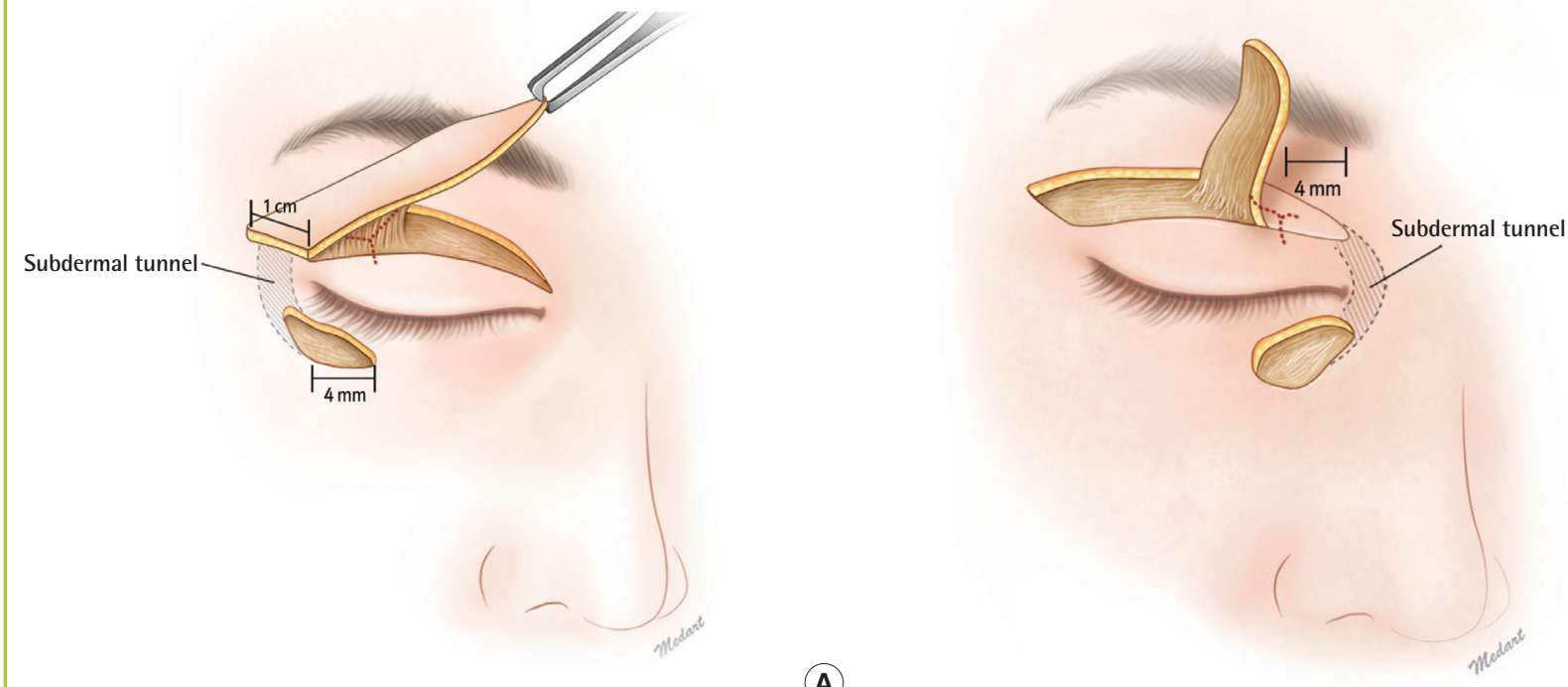

Fig. 2. A case involving the use of a medially based myocutaneous flap

(A) A skin defect $(10 \times 9 \mathrm{~mm})$ after excision of basal cell carcinoma. (B) Elevation of a medially based myocutaneous island flap from the ipsilateral upper eyelid. (C) Immediate postoperative view. (D) View of neutral gaze at 6 months after surgery. (E) View of eye closure at 6 months after surgery.
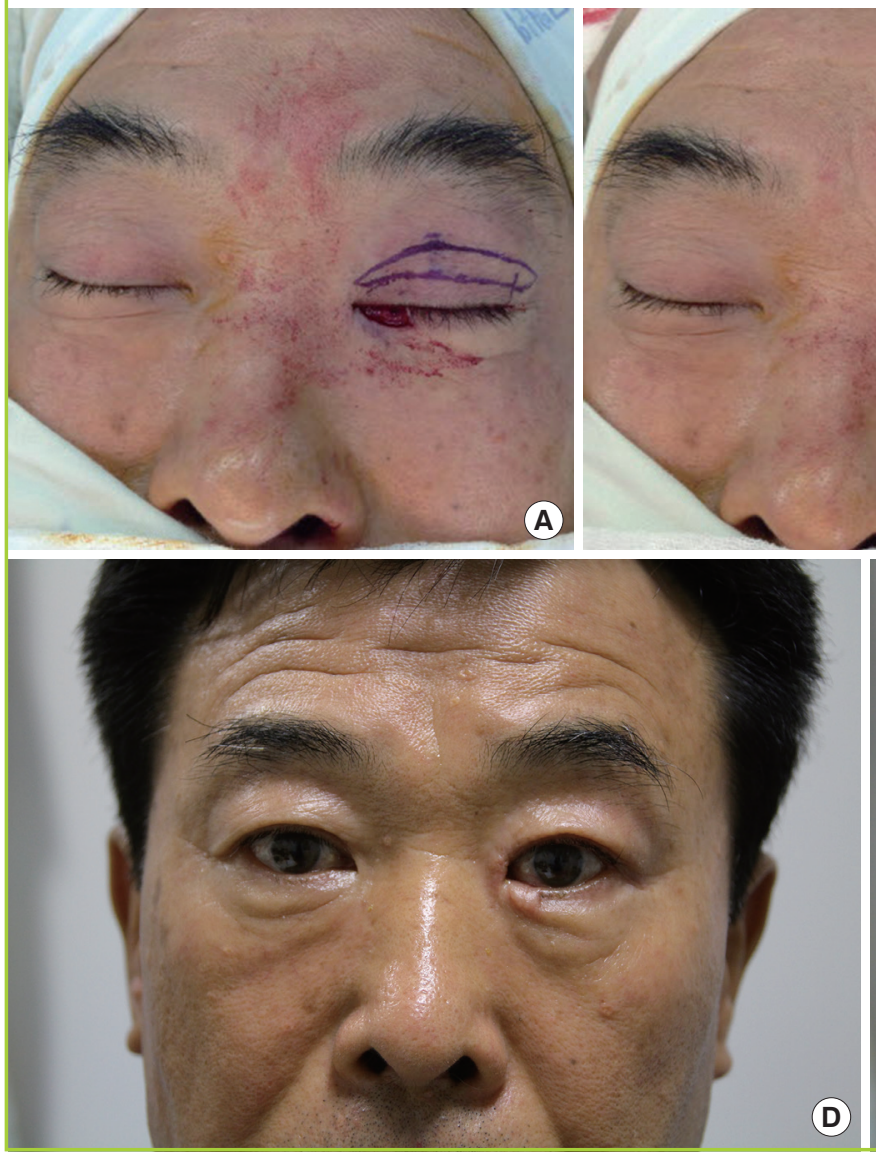
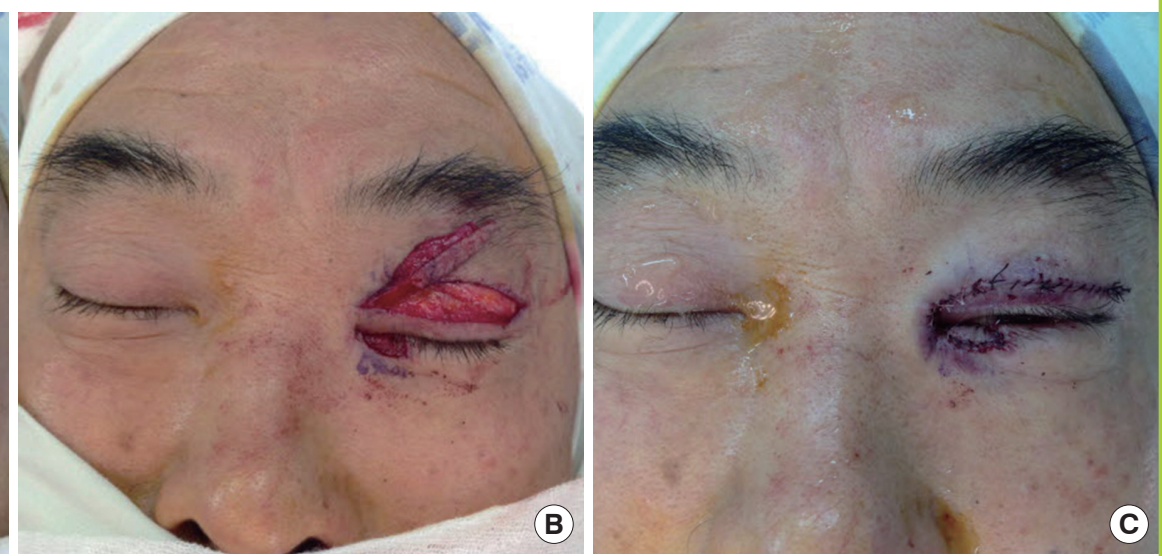

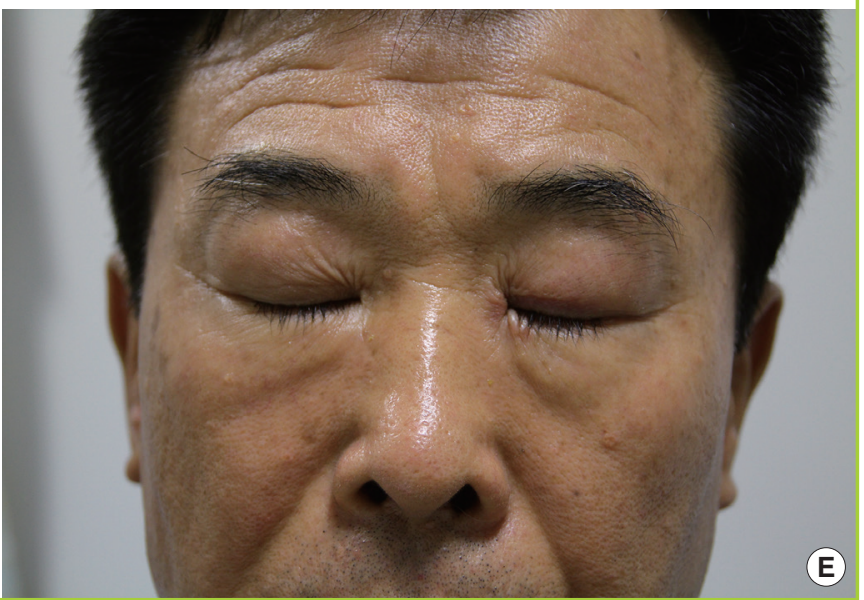




\section{Fig. 3. A case involving the use of a laterally based myocutaneous flap}

(A) A skin defect $(25 \times 7 \mathrm{~mm})$ after excision of basal cell carcinoma. (B) Elevation of a laterally based myocutaneous island flap from the upper eyelid. (C) Subdermal tunneling of the flap to the site of the defect. (D) Immediate postoperative view. (E) View at 6 months after surgery.
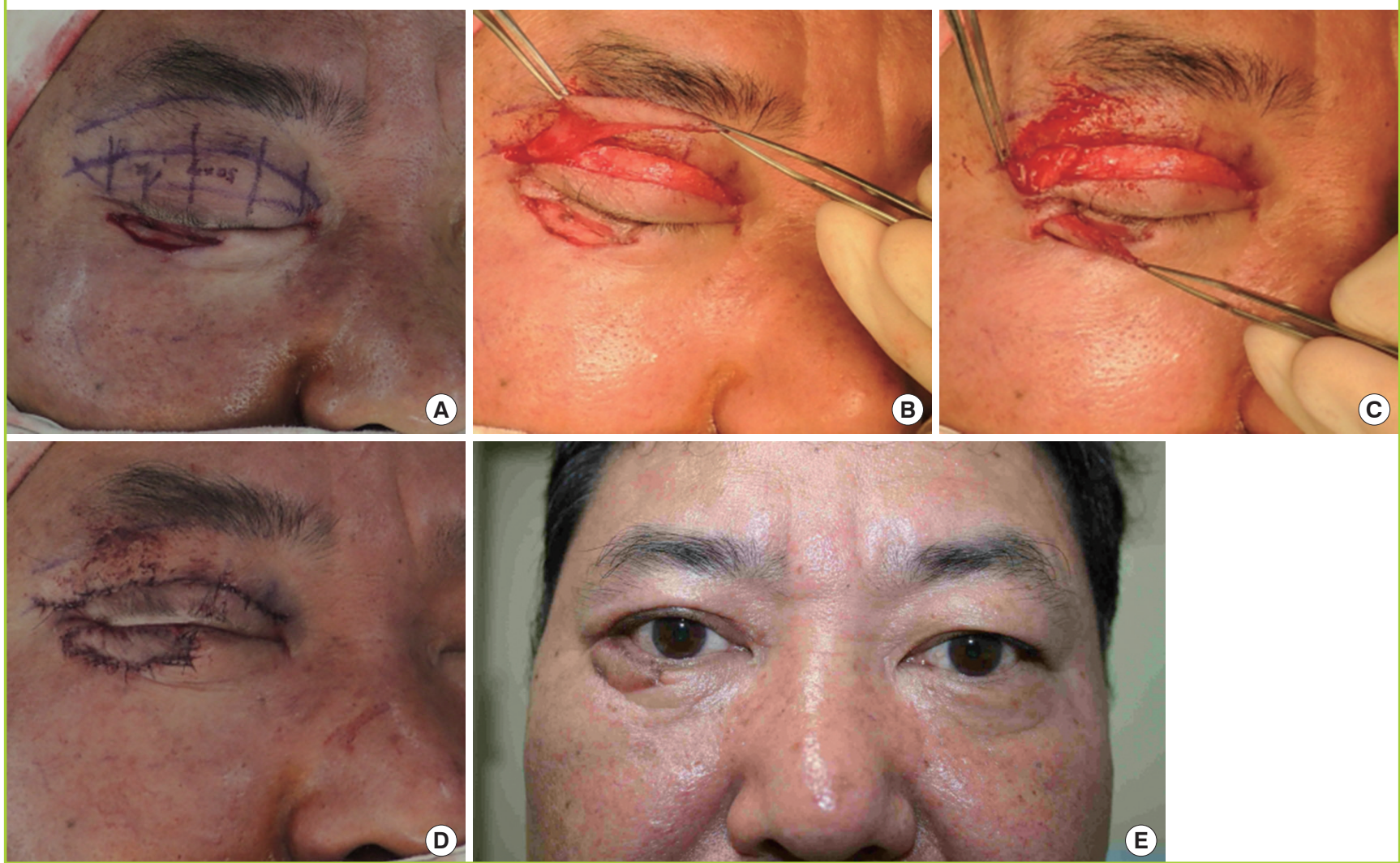

entire flap loss. The average follow-up period for these patients was approximately 10 months. Partial necrosis of the flap was noted in 1 patient who was a heavy smoker at the time of the procedure, but secondary intention healing yielded acceptable aesthetic results. The flaps in general showed some degree of congestion during the first 3-5 days after surgery, but this resolved completely by postoperative days $7-10$. Donor site morbidity was not observed in any case, and normal eyelid movement and eye closure were maintained in all patients. Furthermore, the flaps matched the texture, color, and pliability of the surrounding tissue, and scars were quite inconspicuous. All patients were satisfied with their results.

\section{DISCUSSION}

The medial and lateral canthal regions are important because they contribute to both the normal function of the eye and first impression of a person. Defects in this area can be caused by either trauma or skin malignancies, such as basal cell carcinomas; $14 \%$ of cases of basal cell carcinoma occur in the periorbital area [2]. The elderly population is especially vulnerable to skin tumors of the face, due to the cumulative effects of ultraviolet radia- tion exposure and lack of education about the importance of sunscreen use. Although these skin tumors are easily detected at an early stage, when they present as small, atypical nevi, the resection margin always extends to the orbicularis oculi muscle layer.

In the canthal area, the orbicularis oculi muscle and other fascial structures are delicately agglutinated and intermingled for protection of the globe and preservation of the lacrimal system. Restoration of the color, texture, and thickness of this soft tissue is essential in such a functionally and aesthetically crucial-albeit small—area. Inappropriate reconstructive methods can lead to complications such as epiphora, telecanthus, visibly unnatural appearance, and lowered self-esteem.

Reconstructive methods following skin cancer resection of the canthal regions are selected according to both size and location of the defect and morbidity of the donor site. The simplest method involves the application of a skin graft to the area of the defect. However, the end result of such grafts is often a patchy appearance, due to mismatching color and texture and shrinking of the graft. In addition, when tumor excision leaves the underlying bone or tendon exposed, a skin graft is not a viable option. One flap design used in the reconstruction of the medial canthal region is a glabellar flap, which is designed along the ver- 
tical lines of the glabella. The main disadvantage of this approach is the potential for discrepancy in donor and recipient skin thickness, which may result in a moderate trapdoor deformity [3]. An alternative option is V-Y advancement from the nasal bridge, but use of this method is limited to reconstructing small defects [4].

Another option that uses adjacent tissue is a superiorly based bilobed flap, elevated from the nasal dorsum for medial canthal reconstruction. This technique, first introduced by Perry and Taban [5], took advantage of the redundant vertical tissue of the nasal dorsum, which contains relatively few skin appendages and little subcutaneous fat. However, out of 17 patients in Perry and Taban's [5] study, two with larger defects developed mild ectropion, indicating that this method has certain limitations. Kim et al. [6] proposed the use of an ipsilateral paramedian forehead flap, based on the supratrochlear artery, to cover large defects of the medial canthal region. Although this method has proven to be successful in reconstructing large defects, the resulting bulkiness and potential for telecanthus cannot be ignored. In addition, sensory loss in the forehead is possible, and a skin graft may be required to resurface the donor site [6]. For the lateral canthal area, regional transposition flaps from the upper eyelid, temple, or cheek can be used. However, tube-like or trapdoor scarring may occur, and the results are not aesthetically ideal.

The arterial supply of the palpebral portion of orbicularis oculi muscle is comprised of two arcades, the marginal and the peripheral, which follow the direction of the muscle fibers. These are formed laterally by the communicating branches of the lacrimal artery, and medially by the medial palpebral artery (Fig. 4). As reported in previous studies, a myocutaneous flap based on

\section{Fig. 4. Vascular supply of the eyelids}

Musculocutaneous perforators arising from the peripheral arcade on the upper eyelid tarsal plate can be used as pedicles for the orbicularis oculi myocutaneous island flaps.

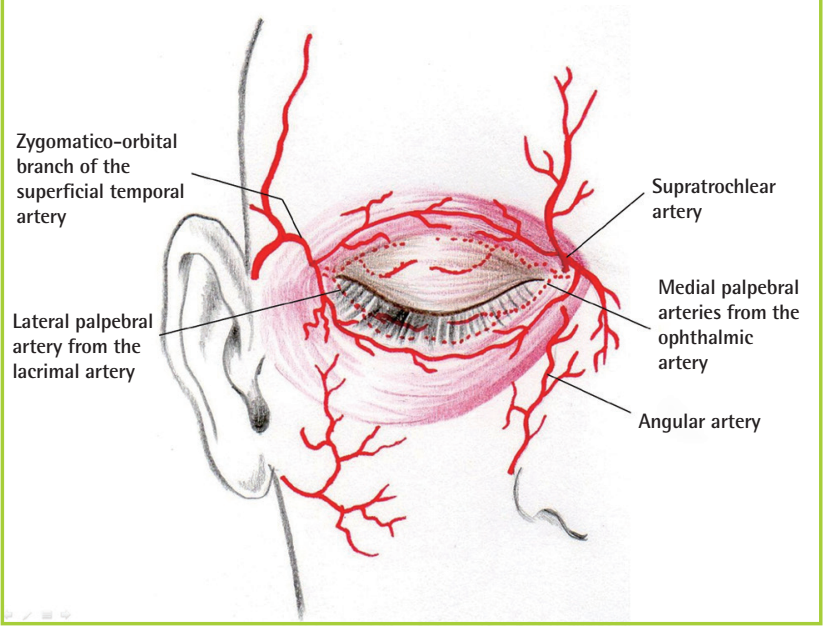

the muscular perforator of the peripheral arcade can be raised as a medially or laterally based flap $[7,8]$. Kim et al. [9] used a Doppler probe to identify the perforators penetrating the muscle from the arcades. We also used a Doppler probe to locate the pedicle at an approximately 4-mm distance from either the medial or lateral canthus in our cases. Because skeletonizing the pedicle may compromise blood flow, it could not be visually recognized with adequate clarity. The resultant $0.5-1-\mathrm{cm}$-wide muscle pedicle, including the perforator, was similar in width to the muscle pedicle used by Porfiris et al. [7] in lower eyelid reconstruction [8]. Although the orbicularis oculi muscle has a rich vascular supply, further detailed anatomical studies of perforator location are necessary for a more reliable elevation of this myocutaneous flap. Another potential benefit is that the flap, with its large rotation arch, can theoretically be rotated 360 degrees to cover the defects located in the periorbital regions, and even in the forehead.

Furthermore, an exceptional advantage of this flap-which is not offered by any other reconstructive option-is the similarity in texture, color, and thickness to the surrounding area. Consequently, the flap can reconstruct defects of many different shapes and quite large sizes—up to $20 \times 17 \mathrm{~mm}$ (Table 1). The flap is raised as a long, leaf-like structure, and can be easily tailored to fit the shape of a particular defect. The flap is both versatile and transferrable to either the medial or lateral canthal area via a corresponding pedicle. In cases where the defect extends further from the medial canthal region to the nasal dorsum, medially based myocutaneous flaps from both upper eyelids may be considered [1]. Local anesthesia is adequate during all procedures, even for aged patients who have medical comorbidities.

The medially based orbicularis oculi myocutaneous flap is a superior option when compared to other reconstructive methods, especially when the punctum or lacrimal apparatus is sacrificed during tumor resection. The lacrimal apparatus can be successfully reconstructed while the soft tissue defect is resurfaced. In contrast to the application of a skin graft to the medial canthal region, the use of a local flap for reconstruction can be beneficial for preventing both contracture of the punctum and distortion of the lacrimal apparatus. In two of our cases, an ophthalmologist performed lacrimal duct reconstruction in conjunction with the orbicularis oculi myocutaneous island flap operation; as a result, normal tear drainage function was restored.

Reconstruction of the canthal regions with an upper eyelid myocutaneous flap has been successfully performed on elderly patients of Western countries $[1,8,10]$, but has not yet been reported in patients from East Asian countries. Regardless of the anatomical differences in the upper eyelid between the two populations - for example, the presence or absence of an upper 
eyelid crease-we found that the orbicularis oculi myocutaneous flap technique can also be appropriate for elderly East Asian patients with redundant upper eyelid skin. East Asians often voluntarily elect to resect this redundant skin, which is a byproduct of aging, as part of a rejuvenating operation [11]. The use of the loose skin is advantageous, as it results in negligible donor site morbidity. However, the applicability of this method may be limited by the amount of excess eyelid skin available. Although the patients in our study had defects that were, on average, smaller than those of Western patients who underwent similar reconstructions, skin tumors involving the eyelid—such as squamous and basal cell carcinomas-are typically detected at an early stage, as mentioned above. This was also the case in our patients, and a larger donor site was unnecessary. In addition, because periorbital tumors usually occur in elderly individuals with loose upper eyelid skin, patients requiring canthal area reconstruction after skin malignancy resection are generally appropriate candidates for this method. We acknowledge that performing reconstruction on one side may lead to asymmetry. However, none of our patients were willing to have an upper blepharoplasty performed on their contralateral side. In cases where the patient wishes to have excess eyelid skin on the contralateral side resected for purposes of both symmetry and aesthetic concerns, the surgeon may elect to do so. Middle-aged patients requiring canthal area reconstruction after traumatic avulsion injury may also be good candidates for this technique, provided that their defects are relatively small compared to the amount of upper eyelid skin available.

\section{REFERENCES}

1. Jelks GW, Glat PM, Jelks EB, et al. Medial canthal recon- struction using a medially based upper eyelid myocutaneous flap. Plast Reconstr Surg 2002;110:1636-43.

2. Shanoff LB, Spira M, Hardy SB. Basal cell carcinoma: a statistical approach to rational management. Plast Reconstr Surg 1967;39:619-24.

3. Field LM. The glabellar transposition "banner" flap. J Dermatol Surg Oncol 1988;14:376-9.

4. Moretti EA, Gomez Garcia F. Myocutaneous flap (V-Y design) from the nasal bridge for medial canthal reconstruction. Ophthal Plast Reconstr Surg 1998;14:298-301.

5. Perry JD, Taban M. Superiorly based bilobed flap for inferior medial canthal and nasojugal fold defect reconstruction. Ophthal Plast Reconstr Surg 2009;25:276-9.

6. Kim JH, Kim JM, Park JW, et al. Reconstruction of the medial canthus using an ipsilateral paramedian forehead flap. Arch Plast Surg 2013;40:742-7.

7. Porfiris E, Georgiou P, Popa CV, et al. Island orbicularis oculis myocutaneous flap from the upper eyelid for lower eyelid reconstruction. Eur J Plast Surg 1998;21:246-8.

8. Tirone L, Schonauer F, Sposato G, et al. Reconstruction of lower eyelid and periorbital district: an orbicularis oculi myocutaneous flap. J Plast Reconstr Aesthet Surg 2009; 62:1384-8.

9. Kim SW, Han HH, Jung SN. Orbicularis oculi myocutaneous island flap for upper eyelid reconstruction. J Craniofac Surg 2012;23:746-8.

10. Stagno d'Alcontres F, D'Amico E, Colonna MR, et al. The orbicularis oculi myocutaneus flap in the repair of the medial canthal region. A new strategy for canthal resurfacing. $\mathrm{Br} \mathrm{J}$ Plast Surg 2004;57:540-2.

11. McCurdy JA. Upper lid blepharoplasty in the Oriental eye. Facial Plast Surg 1994;10:53-66. 